\title{
XXII. Thermal transpiration and radiometer motion
}

\section{Osborne Reynolds}

To cite this article: Osborne Reynolds (1897) XXII. Thermal transpiration and radiometer motion, Philosophical Magazine Series 5, 43:261, 142-148, DOI: 10.1080/14786449708620973

To link to this article: http://dx.doi.org/10.1080/14786449708620973

曲 Published online: 08 May 2009.

Submit your article to this journal $\pi$

Џ Article views: 5

Q View related articles $\sqsubset$ 
and the heat involves the diversion of part of the energy into the forms of radiation, convection, \&c., in fact into other forms than wave propagation.

4. The fourth condition is that the wave-surface propagated from each physical centre within the medium be one which can be represented by an equation of which the parameters are linear quantities of the form $a t, b t, \& c$., where $a, b, \& c$. , are constant velocities. This obviously includes the wavesurface with two or three sheets in crystals, as well as the spherical wave-surfaces of monotropic media. It also includes such waves as are thrown off by an advancing missile, whether in a monotropic or crystalline medium-a kind of wave which probably exists in the æther: and innumerable others. In fact, the theorem is one of perfect generality, and makes it possible physically to resolve any motion however complex which pervades a given space into trains of absolutely uneform plane waves, provided the four conditions enumerated above are fulfilled. The second and third of these conditions are equivalent to saying that the displacements must be such as are not affected by the second order of differential coefficients, and that in general external forces must not intervene. These conditions are essential where the motion is a real physical one, and where the resolution is intended to be into real physical waves. But if the problem be merely kinematical they need not be attended to. This corresponds to the similar statement that has to be made in regard to applications of Fourier's theorem, \&c.

8 Upper Hornsey Rise, I am, Gentlemen, London, N.
1897, January 16.

Yours very faithfully. G. Johnstone Stoney.

XXII. Thermal Transpiration and Radiometer Motion. By Osborne Reinolds:.

JN Yart I. of a paper, contributed to the Phil. Mag. for November 1896 , by Mr. W. Sutherland, there are references (pp. 373, 374) to my paper "On certain Dimensional Properties of Matter in the Gaseous State" (Phil. Trans. R.S. part ii. 1879).

$t$, and provided it do not disturb the uniformity of the plane waves. These requirements are approximately fulfilled in air, so that the theorem applies approximately to sound waves in air, which means that the plane waves into which sound waves in air may be resolved are approximately uniform.

- Communicated by the Author. 
After most flattering expressions respecting the importance and the effect of this paper, followed by severe comments on its mathematical form, Mr. Sutherland says :-

"But what appears to me to be the fatal objection to Reynolds's mathematical method, is that it takes the mind away from definite physical concepts of the actual operation of the causes of thermal transpiration and radiometer motion; and the object of the present paper is to construct a theory of these that will fall into line with the current kinetic theory of gases and keep the physics of the phenomena to the fore."

After this Mr. Sutherland proceeds to describe the foundation on which he has reconstructed the theory.

From his references to my paper, together with the somewhat confusing and sketchy preamble to the definite expression of his foundation, it may well appear that, except for the mathematical form in which it is expressed, this foundation is really the same as that set forth in my paper. It seems therefore to me to be important to point out that there is not the least connexion between these foundations.

According to my showing the sole cause of thermal transpiration is the lateral action of those bounding surfaces which are parallel to the direction in which heat is being conducted; while Mr. Sutherland expressly excludes the action of these walls (or the walls themselves) from any part in thermal transpiration, which be rests solely on the action of direct conduction of heat.

It may seem that the results obtained in the application of theories so differently founded to any particular case must be different ; but this depends on the object sought.

If, as in my paper, the object is to determine the nature of the action, which results in transpiration, from the kinetic theory of gases the results from the different theories will be different; but if, as is Mr. Sutherland's object, it is merely required to express the results of transpiration " in line with the current kinetic theory of gases," then, if the transpiration he has assumed is the same as that obtained by the true theory, the results will to a certain extent correspond.

There is thus danger of the similarity of the results so obtained being taken as a verification of the soundness of the foundation on which Mr. Sutherland has built his theory, and of confusion being added to the inherent difficulties of the subject.

It bas therefore seemed to me to be important not only to point out the difference of the foundations, but also to show clearly that the foundation on which Mr. Sutherland has constructed his theory has no real existence whatever; being the 
result of a somewhat common and, certainly, historical error in mechanical analysis.

In order to make this clear I will recur somewhat to the history of the kinetic theory of gases from 1860. In that year the Phil. Mag. contained a paper by Maxwell in two parts, the first containing his law of distribution, the law of logarithmicdecrement of inequalities in distribution for rigid particles, and the theory of viscosity, and the second part containing the theories of diffusion and the conduction of heat.

After this there appeared (Pogg. Ann. 1862) a paper by Clausius "On the Conduction of Heat by Gases." This was avowedly in answer to a challenge by M. Jochmann, but in reality for the purpose of correcting Maxwell's somewhat ill-considered theory of conduction and replacing it by another on a sound foundation.

In the first paragraph of the second part of his paper Maxwell says :-

"We have shown, in the first part of this paper, that the motions of a system of many small elastic particles are of two kinds: one, a general motion of translation of the whole system, which may be called the motion in mass; and the other a motion of agitation, or molecular motion, in virtue of which velocities in all directions are distributed among the particles according to a certain law. In the cases we are considering, the collisions are so frequent that the law of distribution of the molecular velocities, if disturbed in any way, will be reestablished in an inappreciably short time; so that the motion will always consist of this definite motion of agitation, combined with the general motion of translation."

According to the assumption in the second sentence quoted, if the gas were in steady condition and at rest, no matter what were the space variations of condition, the molecules would leave a spherical element, taken about a point $P$, after having undergone collision within it, with the same mean characteristics in all directions as if the gas about $\mathbf{P}$ had been uniform. And in this case the molecules would reach a plane with mean characteristics as from uniform gas with the mean characteristics of the gas at the points at whioh they last underwent collision. Maxwell seems to have made an error in his analysis which somewhat obscured the results which should follow from this assumption, otherwise it would have appeared to him (in equation 28) that according to the assumption gas could not be at rest and at the same time conducting heat.

Clausius carefully points out these facts both in a note to art. (6) in his paper and in a note to art. (24). 
The fact that a gas or any body can be at rest, and at the same time be transmitting energy, presented a paradox which caused the property of conduction to be the last of the general properties of matter to receive kinetic explanation. This was not for want of perception of the latent motions of the molecules, but for want of a recognition of the only characteristic in such latent motion, or any motion of agitation, which could canse such transmission-the failure to realize that the only means by which matter can transmit energy while in a mean state of rest is that in which the latent motion considered with respect to any plane consists of two streams of matter crossing the plane from opposite sides, the velocity of the one stream being greater than that of the other, and the ratio of the densities being inversely as their velocities.

Clausius seems to have first recognized this. After reading Maxwell's paper and pointing out his errors, he frames a theory of conduction, the same as Maxwell's in so far as the energy carried across the plane by the molecules is taken to represent the heat conducted, but differing in so far as he recognizes the necessity for a difference in the velocity of the opposite streams, such that their velocities shall be inversely proportional to the densities. And taking into account the principle that the mean velocities of molecules after encounter are the same as before, he infers that the characteristic velocities of the two opposite groups of molecules, leaving an element in the direction in which the temperature varies, are such as they would have, if the gas were uniform, plus a velocity in the direction of the fall in temperature depending on the slope of temperature and the mean path of the molecules.

From this start Clausius develops the theory of conduction for gas at rest, using the condition of rest to define the velocity common to the two groups, through a layer of gas of any definite thickness, but only in the ease of indefinite lateral extension. Thus he only studies the action in one dimension, and he nowhere refers to the existence of any solid surfaces.

Maxwell followed with his classical paper of $1866^{*}$, in which he acknowledges Clausius's corrections, and then obtains the equation of continuity, the equations of motion and of energy, and also the equation for the state of conduction of energy, for gas in three dimensions in terms of the logarithmic-decrements of the inequalities, having obtained the constants for these rates for a particular law of force between the molecules.

These equations are applicable to any varying condition of gas provided there is no discontinuity, but they afford no

* Phil. Trans. Roy. Soc. 1867, p. 49. 
means of taking account of the action of a solid surface on the gas to which it is adjacent.

When the idea of thermal transpiration first presented itself I was acquainted with Maxwell's paper, and through this knew of Clausius's work ; but I had not read his paper and knew nothing of his method, so that I was not aware that ho had already introduced expressions for the inequalities of the opposite groups of molecules in form of a series of ascending powers of the mean path of the molecules, the coefficients being the differential coefficients of the varying characteristics, in the case of varying temperature.

But having formed my conception of the mechanical actions, from which $I$ inferred the existence of the property of thermal transpiration as the consequence of a definitely dimensioned structure when the condition of the gas varied in more than one dimension in space, my object being the study of the dimensional properties which can only be studied in three dimensions in space, I adopted a form of equation which, while perfectly general, admitted of the expression of the inequalities resulting from the variation of any characteristic whatsoever of the gas for any group of molecules distinguished by direction, in series of ascending powers of the mean-range (or parameter of the dimensions of structure) in three dimensions in space.

This mathematical system admits of the consideration of such discontinuity as results when the gas is bounded by a solid surface. Since at points in the gas of which the distances from the surface are small compared with the normal mean-range, the range of any group leaving the surface is limited by the distance of the point from the surface.

The action on which thermal transpiration depends when gas at constant pressure but conducting heat is bounded by a solid surface parallel to the direction of conduction, say $x$, is the sum of the mean components of momentum in the direction $x$ of the two groups of molecules distinguished by the signs of their component velocities parallel to $x$, which are brought up to the surface by their component velocities perpendicular to the surface, say in direction $z$. If the component momenta of these groups are equal and opposite, then the condition of the gas at constant pressure will be steady; but if they are not equal and opposite, and if the corresponding groups leaving the surface have equal and opposite momenta in direction $x$ of the surface, in consequence of the action there will be a tangential force between the surface and the gas. And it thus appears that, although the prime cause of thermal transpiration is that relation between the rates of variation of molecular velocities and density which 
causes conduction, there are also involved the action of the lateral components of motion in bringing the molecules up to the surface, and the action of the surface in equalizing the opposite momenta of the opposite groups.

Bearing these incidents and steps in the history of the kinetic theory in mind the precise errors into which $\mathrm{Mr}$. Sutherland has fallen become evident. In the paragraph beginning in middle of p. 375 he begins : "In the kinetic theory the molecules which are considered characteristic of an element are those which have experienced collision in it."

Then he gives reasons for thinking that the walls of the tube produce no effect on the characteristics of the molecules which rebound from them. And thus having cut away the only cause of thermal transpiration he proceeds :-

"Thus, then, if we do not have to take account of reflexion from the walls of the tube, we can consider the gas in it as part of an indefinite mass such that the temperature throughout a plane perpendicular to the axis is the same as that in the section of the tube made by the plane. We wish to find the number of molecules crossing any section of the tube. This is done by Clausius in his theory of conduction in gases, and with greater refinements of accuracy by Tait; but for the sake of clearness we will make the calculation here to a degree of accuracy suitable for present requirements."

It is to be noticed here that he has not stated whether or not the infinite mass of gas is at rest or in motion with respect to the plane; but in the next paragraph he proceeds to consider the quantity of matter carried across the plane by each of the opposite groups of molecules, having respectively the mean characteristics of uniform gas at rest in the mean condition of the actual gas at a distance from the plane on either side equal to the half of the mean component of the free paththus defining the gas as at rest; and at the same time showing that he has in no way realized the point of Clausius's paper, which he quotes-showing, in fact, that he cannot even have read it. Having thus fallen into the error from which Clausius released Maxwell, the inevitable result is that he arrives at the absurd conclusion that in a gas at rest more molecules cross the fixed plane from the cold side than from the hot side. But instead of recognizing the absurdity of this conclusion, he says-" which anounts to the same thing as if the gas had a velocity," and then proceeds to make this unfounded and self-induced velocity the foundation of thermal transpiration, introducing a hypothetical traction between the gas and the solid surface, the effect of which he had previously shown to be nothing.

As shown (art. 88) in my paper, the velocity thus erro- 
niously attributed as an inherent property to gas conducting heat is exactly the velocity which would result from the action of the walls of the tube if the motion of the gas were unrestricted, and hence the danger that the error in Mr. Sutherland's theory might pass unnoticed.

That in adopting this erroneous foundation Mr. Sutherland is not quite satisfied is shown in the last paragraph, p. 374 . This begins :- "The most convenient starting-point is the laws discovered by Clansius (Pogg. Ann. cxv. 1862) for the conduction of heat in gases. In a vertical cylinder of gas, bounded by a solid wall impermeable to heat and two conducting plane ends, the lower at a temperature $\theta_{1}$ the higher at the temperature $\theta_{2}$, when the flow of heat has become steady and the pressure throughout the cylinder is contant, \&c." Clausius expressly excludes solid walls.

Then, again, in the next sentence:- "Now in the establishment of the law of temperature it was shown by Clausius that in a mass of gas which is not uniform in temperature there is motion of the gas in the direction of variability ; but it is assumed (as can easily be proved) that under ordinary circumstances this motion can never produce an appreciable departure from uniformity of pressure, \&c."

This is a complete misstatement. Clansius showed that the velocity which would otherwise arise from the excess of the rate of variation of density over the opposite rate of variation of the velocity of the molecules is balanced by the difference in the opposite velocities of the molecules emerging from the hot and cold sides of an element of the varying gas respectively, the excess being in the direction of the colder side ; but he never mentions a velocity of the gas except as zero. And Mr. Sutherland does not notice that the velocity resulting from the difference just mentioned is towards the colder side, and is opposite to that which he has found, which is towards the hotter side.

In pointing out these errors, although my chief object has been to prevent unnecessary confusion in this abstruse subject, it has also been done with a view to assist Mr. Sutherland in his effort (with which I sympathise). And I hope he will now see that the difficulties of the subject do not exist in mathematical form, but in the conception of the mechanical actions involved; and after mastering these, that he may bo able to produce a translation from the general analysis into a group of particular examples treated in one dimension. For I have no doubt that the great difficulty which many find in this subject arises from unfamiliarity with the expressions in the analysis in three dimensions-a difficulty which is not likely to be removed. 